\title{
GSP07
}

\section{The Value of Integrated Pre-job Modeling and Planning in the Change to Horizontal Drilling and Completion Strategies}

\author{
Ossama Onsy* (Schlumberger)
}

\section{SUMMARY}

is not common practice as a common place drilling strategy nowadays. As the knowledge of horizontal drilling it is not just a matter of drilling, we and the client decided to approach the project as an integrated project in order to be successful for the service company and our client. We found out that it is not enough to steer and place the horizontal well in the reservoir sweet spot. It is recommended to do an integrated pre-job model and full study, starting by the well trajectory stage to completion stage in order not only to drill and geosteer successful the horizontal well but to guarantee the optimum well Production over the life of the well. The study prior to drilling a horizontal well may include many steps depending on the needs from client and drilling knowledge. 


\begin{abstract}
Brown fields are seen in many areas around the globe, including Egypt As reservoirs are depleted new drilling and completion strategies are needed. At this point options for horizontal and reentry drilling wells often come into play. In Egypt the country has little experience drilling horizontal wells and they do not yet form part of the common drilling strategy.. As experience has shown horizontal drilling is not just a matter of drilling, and an integrated multidiscipline approach between operator and service companies is needed in order to bring success for both, While steering and placing the horizontal well in the reservoir sweet spot may be considered one measure of success, this paper demonstrates how best production improvements and reservoir management benefits can be obtained when the drilling phase is planned as part of an integrated pre-job model with full reservoir study. This approach starting with the well trajectory stage, through drilling, completion and production not only helps to successfully position the wellbore but to promote the optimum well Production over the life of the well, while mitigating risks in each of these phases.

Depending on the needs of the client and drilling knowledge in the area, the following points were found to be important considerations for developing and using an Integrated Prejob Model in order to deliver the optimum well trajectory, mitigate drilling hazard, run the proper completion, as well as to maintain a high productivity index.
\end{abstract}

1) An Updated Geological model: This need to be verified by seismic data in $3 \mathrm{D}$ is needed in order to visualize the formation dip, layer thickness, offset well locations in respect to the planned well trajectory and faults intersected in the area. This is essential for trajectory and well placement planning.

2) A Geomechanic analysis: This is required to check if wellbore instability-related problems are expected and if yes, to minimize their impact by suggesting the best direction for drilling and optimum mud weights. Moreover, the ratio of Kv.to Kh. driven from core analysis should be considered since it has a direct effect on horizontal lateral drainage, and hence production.

3) Run reservoir management simulator software; perform reservoir sensitivity studies on the effect of different well trajectories (eg.slanted or horizontal) on production. This is especially necessary in the case of two or more thin successive pay zones, to optimize the lateral length, maximize the production, and minimize the water cut. The same model can be applied to simulate the expected production rate and also review cost and performance of the well geometry changes. (vertical, inclined, slanted, horizontal)

4) Best choice of Logging While Drilling (LWD) tool technology to evaluate and steer the well through the reservoir.. This is based on the modeling the tool response to different geological scenarios and petrophysical properties (GR, resistivity, etc) . Reservoir thickness and mud type to be used are important. Dip changes, layer thinning or thickening, etc), can be modeled to understand the tool responses and determine if it will be possible to steer the well in the desired zone.

5) The results of the Integrated Pre-job model need to be considered in order to plan technically and economically sound completion schemes (completion type, mesh size, screen positioning, flow control devices, etc), as well as to identify proper stimulation techniques (acidizing, hydraulic fracturing, diversion, etc.) which will maximize the reservoir response. 
We present 2 wells in 2 different fields which followed two steps of the above methodology prior to drilling for Petrobel in Egypt. In both cases the reservoir sensitivity study and tool response models stages alone led to a change in the client drilling and completion strategy.

In the first case the well was successfully steered in a challenging interbedded geological environment using an LWD Deep Resistivity Distance to Boundary Tool, leading to very good results: An outstanding $93 \%$ net to gross and initial production rate three times the vertical wells rate producing from the same pay zone in the field. These results were in line with the reservoir model prediction. In the second case the well was initially drilled as a deviated pilot. Upon receiving the reservoir sensitivity study results the client decided to change his drilling and completion strategy. A horizontal side track was drilled, also resulting in significant production increase, which was also inline with the reservoir model prediction.

\section{Acklowledgements.}

The authors would like to thank Ahmed Ismail, Moamen Ramadan for their Reservoir Prediction modeling work and Aristides Orlandi Neto his contributions and ideas 